\title{
On the potential of light-by-light scattering for invisible axion detection
}

\author{
D. Bernard \\ LPNHE, Ecole Polytechnique, IN2P3 63 CNRS, 91128 Palaiseau, France \\ Submitted to Il Nuovo Cimento A
}

September 3, 1997

\begin{abstract}
An experimental program is in progress, searching for possible non QED signals in elastic photon-photon scattering in the optical domain[1]. We examine here the potential of this process for the detection of a possible light pseudoscalar such as the axion.
\end{abstract}

\section{A Introduction}

The standard model currently provides a consistent description of elementary particles and of their interactions. Yet, it contains spontaneously broken global symmetries which imply the existence of Goldstone bosons. In the case of the U(1) Peccei-Quinn symmetry introduced to explain the absence of CP violation in QCD [2], this particle is called the axion. These particles have escaped detection up to now.

Originally, the mass scale of the $\mathrm{U}_{P Q}(1)$ symmetry breaking was thought to be the same as the one for weak interactions, which implies an axion mass $m_{a} \approx 100 \mathrm{keV}$. This type of axion has been ruled out by particle decay and beam dump experiments. For this reason, axions with smaller mass and weaker coupling to matter, called invisible axions, have been considered. Various types of axions have been considered. The KSVZ axions[3] are hadronic axions with only induced coupling to leptons. The DFSZ axions[4] naturally couple to all fermions. They couple to two photons via the anomaly of the triangular diagram (fig. 1).

In these models, the mass of the axion $m_{a}$ and its coupling constant to two photons $g$ are proportional to each other, with :

$$
m_{a}=g \times C
$$

Where $[5] C_{\mathrm{KSvz}}=2.7 \cdot 10^{18} \mathrm{eV}^{2}$ and $C_{\mathrm{DFSz}}=7.4 \cdot 10^{18} \mathrm{eV}^{2}$.

Pseudoscalar or scalar particles that couple to two photons but not to fermions have also been considered in ref. [6]. In that case, $m_{a}$ and $g$ are two independent parameters.

The actual window for the invisible axion is mainly limited by astrophysical considerations (see the reviews in [7]). Axions can be produced in the core of stars via the Primakoff 


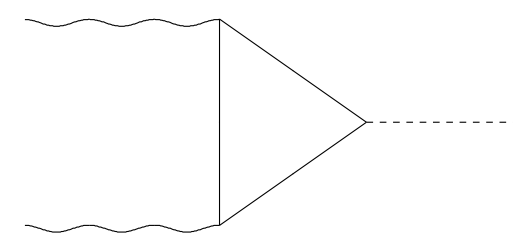

Figure 1: Axion coupling to two photons.

process. Due to their low interaction with matter, these particles escape almost freely. The star contracts and increases its central temperature to compensate for this additional energy loss. The life-time of the star is then reduced, which also reduces the number of observed stars in the given part of the sequence. In the case of the KSVZ axion, a limit of the coupling constant is obtained from the helium burning stars $g<3.10^{-10} \mathrm{GeV}^{-1}$, corresponding to $m_{a}<2 \mathrm{eV}[8]$. A more stringent mass upper limit is obtained from the observation of the neutrino pulse from SN1987A. Axions with masses in the range $10^{-3} \mathrm{eV}<m_{a}<2 \mathrm{eV}$ would have accelerated the cooling of the core and shortened the neutrino burst [9]. A lower bound of the mass is obtained from cosmology, as axions with a mass lower than $10^{-6} \mathrm{eV}$ would close the universe [10].

The window for the invisible axion is therefore $10^{-6}-10^{-3} \mathrm{eV}$ (a recent discussion is given in [11]). In the lower part of the allowed mass range, axions may constitute a part of the dark matter in the universe.

A less stringent limit, but free from any astrophysical model, of $g<3.6 \cdot 10^{-7} \mathrm{GeV}^{-1}$ (for $m_{a}<10^{-3} \mathrm{eV}$ ) has been obtained recently by the study of the propagation of a laser beam through a transverse magnetic field [12]. The experiment searched for the birefringence induced by the axion coupling to two photons. This limit is expected to improve by a factor 40 with the PVLAS experiment which is in preparation [13].

In this Letter, we examine the potential of axion discovery in the elastic scattering of photons. An experiment searching for possible non QED signals in photon-photon elastic scattering in the optical domain has been performed recently by colliding two high power pulsed lasers beams [1]. The obtained upper limit of the cross section is $10^{-39} \mathrm{~cm}^{2}$ and is expected to improve by 20 orders of magnitude in an experiment which is in preparation, with the use of high repetition rate lasers, and by stimulating the reaction with a third beam [1]. 


\section{B Scattering cross section}

The interaction of the axion with two photons is described[14] by the interaction Lagrangian $\mathcal{L}_{I}$ :

$$
\mathcal{L}_{I}=\frac{g}{4} \Phi F^{\mu \nu} \tilde{F_{\mu \nu}}
$$

Here $\Phi$ is the axion field, $\tilde{F_{\mu \nu}}=\frac{1}{2} \epsilon_{\mu \nu \lambda \sigma} F^{\lambda \sigma}$ is the dual of the electromagnetic field tensor. The derivative coupling to the electromagnetic field, together with the smallness of the coupling constant, yields a very small coupling of the axion to two photons. From the antisymmetry of $\epsilon_{\mu \nu \lambda \sigma}$, one obtains :

$$
\mathcal{L}_{I}=\frac{g}{2} \Phi \partial^{\nu} A^{\mu} \epsilon_{\mu \nu \lambda \sigma} \partial^{\sigma} A^{\lambda}
$$

The decay rate of an axion in two photons is obtained from the expression of $\mathcal{L}_{I}[7]$ : $\Gamma_{\gamma \gamma}=g^{2} m_{a}^{3} / 64 \pi$.

We now turn to $\gamma \gamma$ elastic scattering. The differential cross section in the center of mass system $(\mathrm{cms})$ reads :

$$
\frac{\mathrm{d} \sigma}{\mathrm{d} \Omega}=\frac{|\mathcal{M}|^{2}}{64 \pi^{2} s}
$$

where the matrix element $\mathcal{M}$ is the sum of the terms obtained from the two diagrams in figure $2: \mathcal{M}=\mathcal{M}_{a}+\mathcal{M}_{b}$, and $s$ is the $c m s$ energy. $\mathcal{M}_{a}(t$ channel $)$ and $\mathcal{M}_{b}$ (s channel) are obtained from the expression of $\mathcal{L}_{I}$ :

$\mathcal{M}_{a}=\frac{-i g^{2}}{4} \frac{\left(\epsilon_{\mu \nu \lambda \sigma}{k^{\prime}}_{1}^{\prime} k_{1}^{\sigma} \epsilon_{1}^{\prime \mu} \epsilon_{1}^{\lambda}\right)\left(\epsilon_{\mu \nu \lambda \sigma} k_{2}^{\nu}{k_{2}^{\prime}}_{2} \epsilon_{2}^{\mu} \epsilon_{2}^{\prime \lambda}\right)}{\left(k_{1}-k_{1}^{\prime}\right)^{2}-m_{a}^{2}}+\left(k_{1} \leftrightarrow k_{2}\right)+\left(k_{1}^{\prime} \leftrightarrow k_{2}^{\prime}\right)+\left(k_{1} \leftrightarrow k_{2}\right.$ and $\left.k_{1}^{\prime} \leftrightarrow k_{2}^{\prime}\right)$

$\mathcal{M}_{b}=\frac{-i g^{2}}{4} \frac{\left(\epsilon_{\mu \nu \lambda \sigma} k_{1}^{\prime \nu} k_{2}^{\prime \sigma} \epsilon_{1}^{\prime \mu} \epsilon_{2}^{\prime \lambda}\right)\left(\epsilon_{\mu \nu \lambda \sigma} k_{1}^{\nu} k_{2}^{\sigma} \epsilon_{1}^{\mu} \epsilon_{2}^{\lambda}\right)}{\left(k_{1}+k_{2}\right)^{2}-m_{a}^{2}}+\left(k_{1} \leftrightarrow k_{2}\right)+\left(k_{1}^{\prime} \leftrightarrow k_{2}^{\prime}\right)+\left(k_{1} \leftrightarrow k_{2}\right.$ and $\left.k_{1}^{\prime} \leftrightarrow k_{2}^{\prime}\right)$

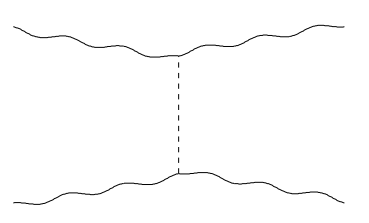

a

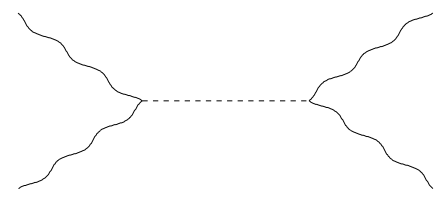

$\mathrm{b}$

Figure 2: Photon-photon elastic scattering through the exchange of an axion. a) : $t$ channel, b) : s channel.

Here $k_{i}, i=1,2\left(k^{\prime}{ }_{i}, i=1,2\right)$ denote the four-momenta of the incoming (outgoing) photons, and $\epsilon_{i}, i=1,2$ and $\epsilon_{i}^{\prime}, i=1,2$ denote the corresponding polarisation vectors. 
The four terms in the expression of $\mathcal{M}_{b}$ are equal to each other. The four terms in the expression of $\mathcal{M}_{a}$ are equal two by two. We compute the 16 matrix elements $\mathcal{M}_{i j k l}$, with indices 1 for linear polarisation in the plane of scattering, and indices 2 for linear polarisation perpendicular to that plane. We obtain :

$$
\begin{aligned}
& \mathcal{M}_{1122}=\mathcal{M}_{2211}=\frac{i g^{2} k^{2}}{2}\left(\frac{c_{\varphi / 2}^{4}}{c_{\varphi / 2}^{2}+m_{a}^{2} / 4 k^{2}}+\frac{s_{\varphi / 2}^{4}}{s_{\varphi / 2}^{2}+m_{a}^{2} / 4 k^{2}}\right) \\
& \mathcal{M}_{1212}=\mathcal{M}_{2121}=-\frac{i g^{2} k^{2}}{2}\left(\frac{2}{-1+m_{a}^{2} / 4 k^{2}}+\frac{s_{\varphi / 2}^{4}}{s_{\varphi / 2}^{2}+m_{a}^{2} / 4 k^{2}}\right) \\
& \mathcal{M}_{1221}=\mathcal{M}_{2112}=-\frac{i g^{2} k^{2}}{2}\left(\frac{2}{-1+m_{a}^{2} / 4 k^{2}}+\frac{c_{\varphi / 2}^{4}}{c_{\varphi / 2}^{2}+m_{a}^{2} / 4 k^{2}}\right),
\end{aligned}
$$

where $\varphi$ is the scattering angle and $c_{\varphi / 2}=\cos (\varphi / 2), s_{\varphi / 2}=\sin (\varphi / 2)$. The 10 other terms are equal to zero. We see that $\mathcal{M}_{1212}$ and $\mathcal{M}_{1221}$ present the usual divergence in the $s$ channel at $s=m_{a}^{2}$.

The polarised differential cross sections in the high energy $\left(k \gg m_{a}\right)$ and in the low energy limit, are plotted in figure 3 .
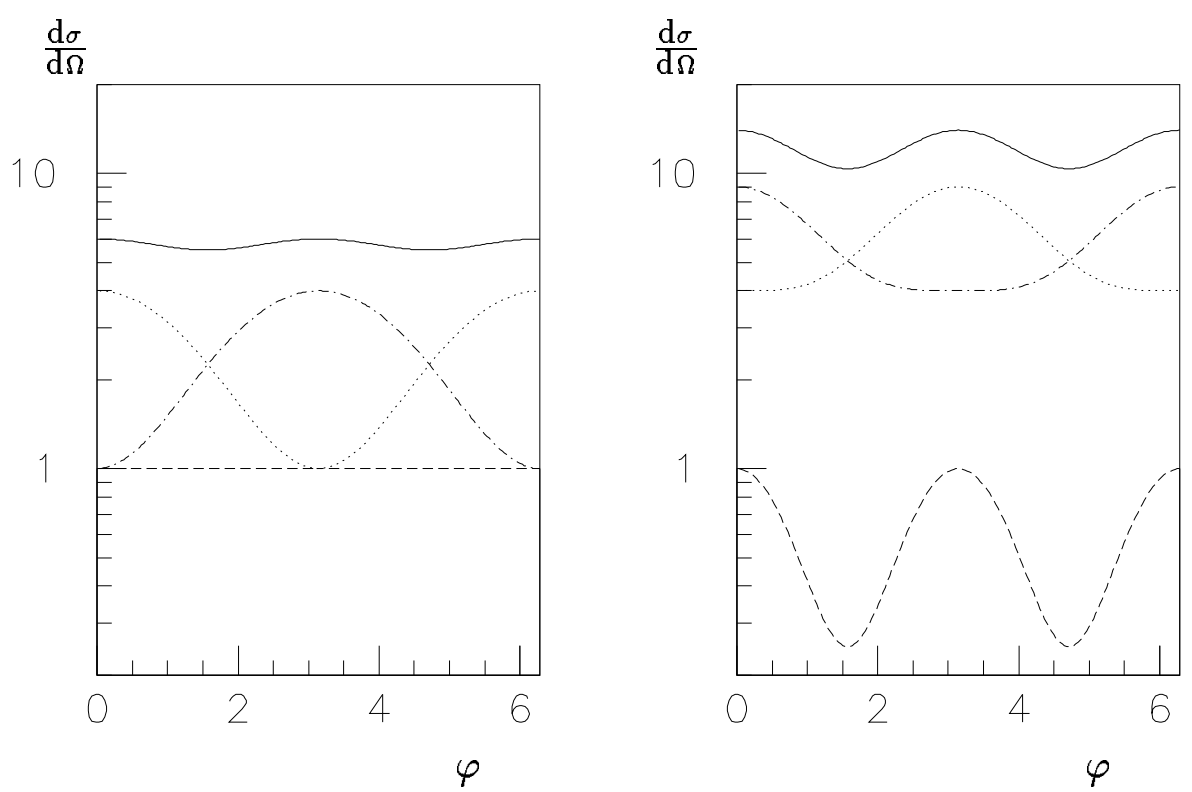

Figure 3: Angular variation of the polarized cross sections, in units of $g^{4} k^{2} / 1024 \pi^{2}$ (high energy, left) and in units of $g^{4} k^{6} / 64 \pi^{2} m_{a}^{4}$ (low energy, right), as a function of the scattering angle. Polarisations 1122, 1212, 1221 are noted respectively by dashed, dotted, and dashed-dotted line. A solid line indicates the unpolarized cross section.

At high energy, the asymptotic behaviour of the polarised total cross-sections is :

$$
\sigma_{1122, \infty}=\frac{g^{4} k^{2}}{256 \pi}, \quad \sigma_{1212, \infty}=\frac{7}{3} \frac{g^{4} k^{2}}{256 \pi}, \quad \sigma_{1221, \infty}=\frac{7}{3} \frac{g^{4} k^{2}}{256 \pi}
$$


and for the unpolarised total cross-section : $\sigma_{\infty}=\frac{17}{3} \frac{g^{4} k^{2}}{256 \pi}$. At low energy we get :

$$
\sigma_{1122,0}=\frac{7}{15} \frac{g^{4} k^{6}}{16 \pi m_{a}^{4}} \quad \sigma_{1212,0}=\frac{83}{15} \frac{g^{4} k^{6}}{16 \pi m_{a}^{4}} \quad \sigma_{1221,0}=\frac{83}{15} \frac{g^{4} k^{6}}{16 \pi m_{a}^{4}}
$$

and for the unpolarised total cross-section : $\sigma_{0}=\frac{173}{15} \frac{g^{4} k^{6}}{16 \pi m_{a}^{4}}$.

\section{Comparison with the QED cross section}

In general the scattering amplitude by the exchange of an axion will interfere with the QED amplitude. Here we only compare the corresponding cross sections. The QED cross section was calculated by De Tollis[15]; for a photon with a $c m s$ energy $\hbar \omega \ll m c^{2}$, where $m$ is the mass of the electron, we have :

$$
\sigma_{Q E D}=\frac{973}{10125 \pi} \alpha^{2} r_{e}^{2}\left(\frac{k}{m c^{2}}\right)^{6}
$$

Here, $\alpha$ is the fine structure constant and $r_{e}$ is the classical radius of the electron. We then get $\sigma_{Q E D}[\mathrm{mbarn}]=7.310^{-39}(k[\mathrm{eV}])^{6}$.

\section{C.1 Low energy limit}

In the low energy limit, $\left(k \ll m_{a}\right)$ the two cross sections grow like $k^{6}$. The dimensionned axion cross section reads :

$$
\sigma_{0}=\frac{173}{240 \pi} \frac{k^{6}}{C^{4}}(\hbar c)^{2}
$$

that is : $\sigma_{0}[\mathrm{mbarn}]=1.710^{-57}(k[\mathrm{eV}])^{6}$ for the KSVZ axion and $\sigma_{0}[\mathrm{mbarn}]=2.310^{-59}(k[\mathrm{eV}])^{6}$ for the DFSZ axion, twenty orders of magnitude below the QED cross section.

The axion contribution would dominate the cross section only for :

$$
C>\left[\frac{173 \times 675}{16 \times 973}\right]^{1 / 4} \frac{\left(m c^{2}\right)^{2}}{\alpha}
$$

that is $C>5.910^{13} \mathrm{eV}^{2}$, five orders of magnitude below the expected value for the $\operatorname{axion}(\mathrm{s})$.

\section{C.2 High energy limit}

For experiments in the visible domain and an axion mass in the allowed window, we are in the high energy regime, $\left(k \gg m_{a}\right)$. The exchange of an axion would dominate for $\sigma_{\infty}>\sigma_{Q E D}$, that is for :

$$
k<\frac{m_{a}}{C} \frac{\left(m c^{2}\right)^{2}}{\alpha}\left[\frac{17 \times 3375}{256 \times 973}\right]^{1 / 4}
$$

that is $k<m_{a} \times(3-9) 10^{-6}$, depending on the nature of the axion. This result is incompatible with the hypothesis of the high energy limit.

Therefore the contribution of the axion never dominates (fig. 4). The soft dependance of the axion cross section and of the QED cross section [16] on polarisation and on angle does not provide any help. 


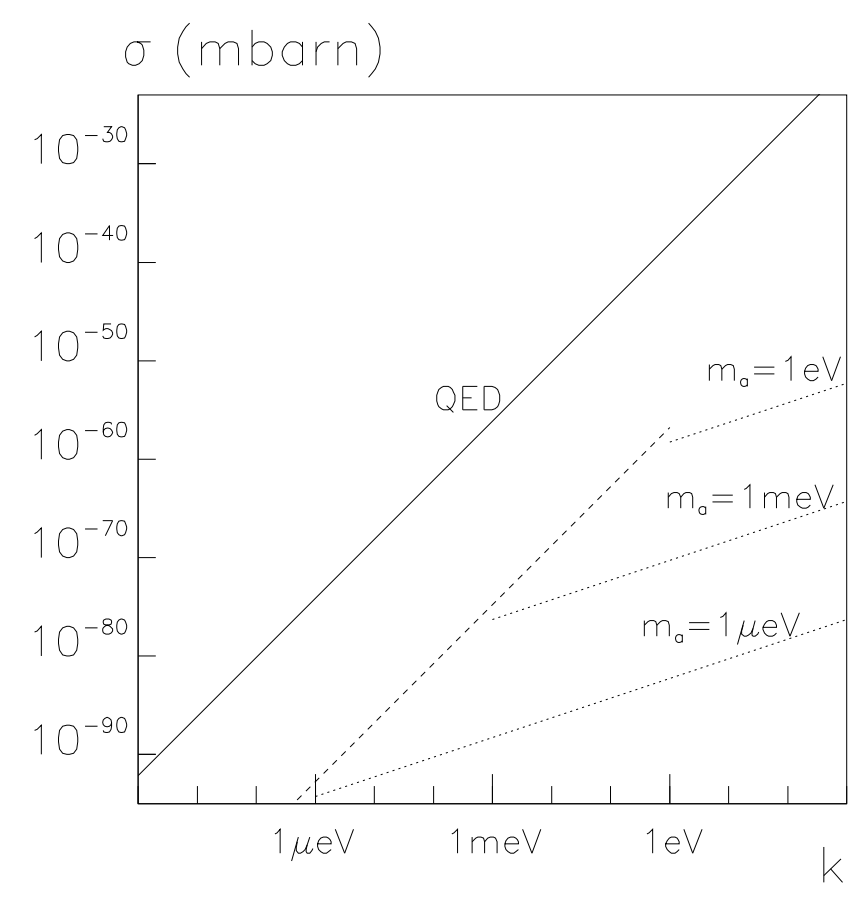

Figure 4: The QED (solid line) and the non-resonant axion cross section (high energy : dotted; low energy : dashed), as a function of the cms photon energy $k$, for various values of the axion mass.

\section{Upper limits on the coupling constant}

In the more general case of a pseudoscalar with independent values of $m_{a}$ and $g$, the upper limit on $g$ is limited by the QED background and is given in the high energy limit by eq. (13), that is :

$$
g>\frac{\alpha k}{\left(m c^{2}\right)^{2}}\left[\frac{256 \times 973}{17 \times 3375}\right]^{1 / 4}
$$

i.e. $g\left[\mathrm{GeV}^{-1}\right]>4.10^{-5} k[\mathrm{eV}]$, only competitive wrt the limit obtained by Cameron et al. [12] for $m_{a}>7 \cdot 10^{-3} \mathrm{eV}$. The upper limit of the elastic cross section obtained in reference [1] is $10^{-12}$ mbarn. If improved by twenty orders of magnitude as expected down to $10^{-32}$ mbarn - it will provide an upper limit on the coupling constant :

$$
g_{\text {lim }}=\left[\frac{3 \times 256 \pi}{17} \frac{\sigma}{(\hbar c)^{2} k^{2}}\right]^{1 / 4}
$$

that is $g_{\text {lim }}=1.410^{-3} \mathrm{GeV}^{-1}$, only.

The limits on the coupling constant of a pseudoscalar to two photons are plotted as a function of its mass in figure 5, inspired by fig. 20 of ref. [12]. A discussion of previous limits and complete references can be found therein.

The value of $g$ that can be attained in a non-resonant photon-photon scattering experiment at $k=1 \mathrm{eV}$, and at the limit of QED background is denoted by NR(QED). The limit that could be obtained in the experiment using a stimulating beam is denoted by $\mathrm{NR}\left(1^{\prime}\right)$. 


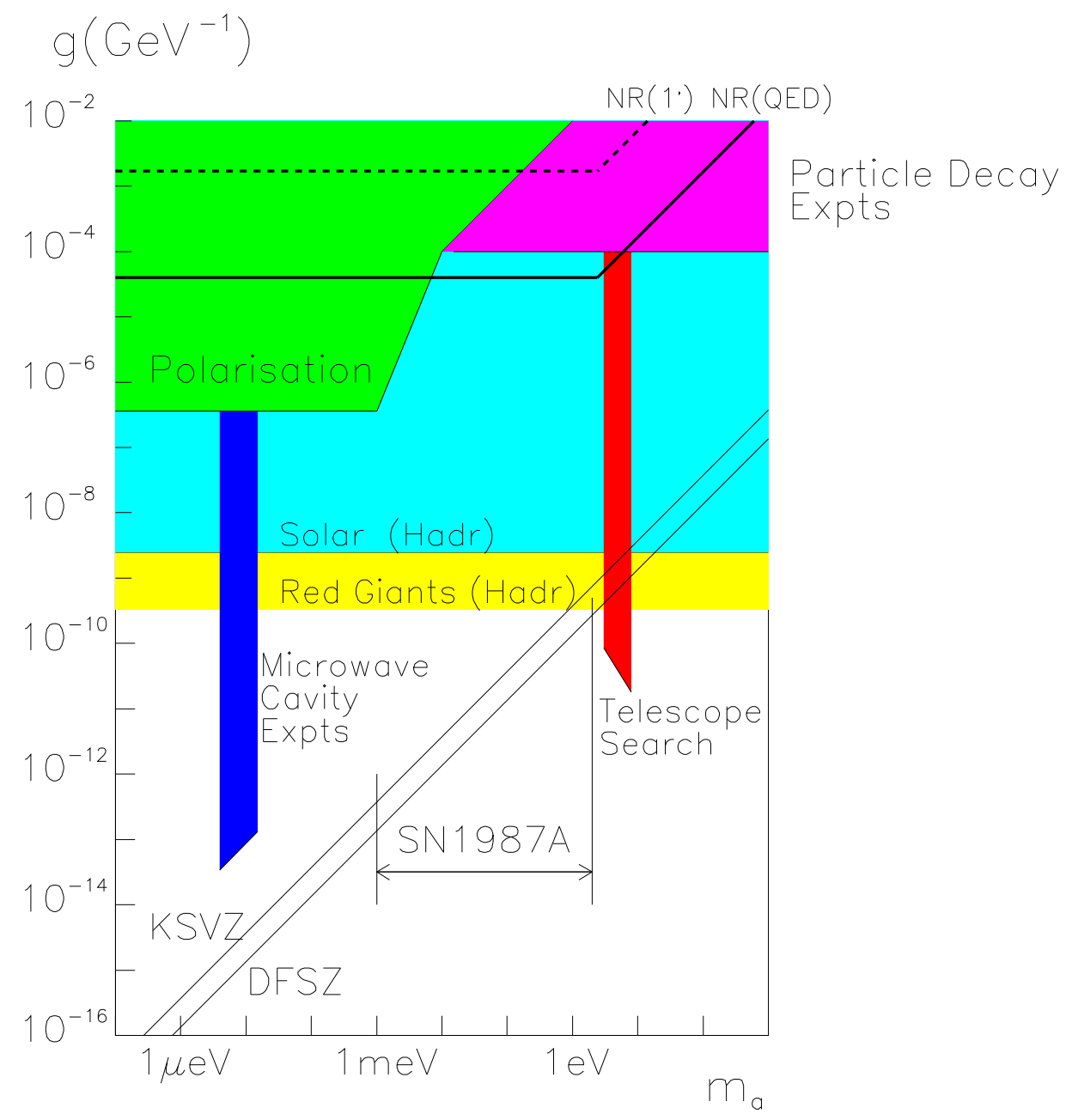

Figure 5: Limits on axion mass and axion coupling to two photons. See text.

The non-resonant photon-photon scattering is clearly of a little interest in the search for a light pseudoscalar.

\section{E Behaviour close to the pôle}

Close to the pôle, $2 k \approx m_{a}$, the cross section is described by a Breit-Wigner formula :

$$
\sigma_{B W}=\frac{\pi}{4 k^{2}} \frac{\Gamma^{2}}{\left(2 k-m_{a}\right)^{2}+\Gamma^{2} / 4}
$$

Its maximum value $\sigma_{B W, 0}=\pi / k^{2}$, is very large, close to $10^{18} \mathrm{mbarn}$ - at the unitary limit - but the width is tiny; $\Gamma=m_{a}^{5} /\left(64 \pi C^{2}\right)$ i.e. $\Gamma[\mathrm{eV}]=(0.9-6.8) 10^{-40}\left(m_{a}[\mathrm{eV}]\right)^{5}$.

One could envisage searching for the resonant exchange in photon-photon elastic scattering, in close analogy with the $\pi^{0}$ production used in ref. [17].

One limitation of this method is due to the finite mass range that can be tested, that is of the spectrum of the $\mathrm{cms}$ energy in the collision. In the experiment described in ref. [1], 
the spectral width of the beams were about $1 \AA$, at a $c m s$ energy of $3.3 \mathrm{eV}$, corresponding to a $\mathrm{cms}$ relative spectral width $\Delta k / k$ of only $0.710^{-3}$ at FWHM.

The useful fraction $f$ of the luminosity is of the order of :

$$
f=\frac{\Gamma}{m_{a}} \frac{k}{\Delta k}
$$

so that the limit on the coupling constant is :

$$
g_{\text {lim }}=\left[16 \frac{\sigma_{l i m}}{(\hbar c)^{2}} \frac{\Delta k}{k}\right]^{1 / 2}
$$

The corresponding value for ref. [1] is $g_{\text {lim }}=1.710^{-7} \mathrm{GeV}^{-1}$, but the tested axion mass range is very narrow, $3.33 \pm 0.001 \mathrm{eV}$.

In the experiment with a stimulating beam which is in preparation, short ( $35 \mathrm{fs})$ pulses are used with $40 \mathrm{~nm}$ spectral width at FWHM, so that the relative spectral width $\Delta k / k$ in the $\mathrm{cms}$ is close to $3.6 \%$. We would then obtain a limit of $g_{l i m}=1.210^{-16} \mathrm{GeV}^{-1}$ at $m_{a}=1.641 \pm 0.025 \mathrm{eV}$. An increased energy range could be tested with given lasers by varying the crossing angle. The exploration of a larger range would require the use of different laser wavelengths (e.g. by frequency multiplying).

Actually the resonant state lives for a time of the order of $\tau=\hbar /(F \Gamma)$, where $F$ is the stimulating factor. If the stimulating beam is diffraction and Fourier limited, and if its optics and pulse length are adapted to the two other colliding beams, $F$ is simply equal to the number of photons in the stimulating beam. For $m_{a}=1 \mathrm{eV}, g=10^{-10} \mathrm{GeV}^{-1}$, $F=10^{16}$, we get $\tau=10^{9}$ s. Clearly the axion does not decay inside the detector.

Asking that the decay time be comparable with the stimulating time, i.e. equal to the pulse length, we obtain a limit on the coupling constant of :

$$
g_{l}=\left[64 \pi \frac{\hbar}{\tau F} \frac{1}{m_{a}^{3}}\right]^{1 / 2}
$$

that is $g\left[\mathrm{GeV}^{-1}\right]=0.02\left(m_{a}[\mathrm{eV}]\right)^{-3 / 2}$ for $\tau=35 \mathrm{fs}$. Clearly the detection of a pseudoscalar coupled to two photons by an appearance stimulated resonant photon-photon scattering experiment is out of reach.

\section{F Conclusion}

At the present time, the closing of the high mass $\left(m_{a}>10^{-3} \mathrm{eV}\right)$ part of the axion window relies on astrophysical arguments. In this range, the limit obtained by direct measurements is provided by decay experiments and amounts to $10^{-4} \mathrm{GeV}^{-1}$.

The potential of improvment in this range by non resonant photon-photon scattering is limited by the QED background; it is marginal and amounts to $4.10^{-5} \mathrm{GeV}^{-1}$.

The resonant scattering, though interesting in principle, is actually indetectable because the intermediate axion does not decay during the stimulating time.

\section{References}

[1] F. Moulin, D. Bernard, F. Amiranoff, Z. Phys. C 72607 (1996). 
[2] R. D. Peccei, H. R. Quinn, Phys. Rev. Lett. 38, 1440 (1978); Phys. Rev. D 161791 (1977);

S. Weinberg, Phys. Rev. Lett. 40223 (1978);

F. Wilczec, Phys. Rev. Lett. 40279 (1978).

[3] J. Kim, Phys. Rev. Lett. 43 (1979) 103;

M. A. Shifman, A. I. Vainshtein and V. Z. Zakharov, Nucl. Phys. B 166 (1980) 493.

[4] M. Dine, W. Fischler and M. Srednicki, Phys. Lett. B 104 (1981) 199;

A. P. Zhinitnisky, Sov. J. Nucl. Phys. 31 (1980) 260.

[5] R. Stroynowski, A. R. Zhitnitsky, preprint SMU-HEP-94-12, Aug. 1994, hep$\mathrm{ph} / 9409251$.

[6] E. Massò, R. Toldrà, Phys. Rev. D 52 (1995) 1755.

[7] J. E. Kim, Phys. Rep. 150 (1987) 1;

M. S. Turner, Phys. Rep. 197 (1990) 67;

G. G. Raffelt, Phys. Rep. 198 (1990) 1.

[8] G. G. Raffelt and D. S. P. Dearborn, Phys. Rev. D 36 (1987) 2211.

[9] M. S. Turner, Phys. Rev. Lett., 60 (1987) 1797;

R. Mayle et al., Phys. Lett. B 203 (1988) 188, 219 (1989) 515.

[10] See the discussion in ref. [8], and the references in [7] therein.

[11] QCD, Strong CP and Axions, R. D. Peccei, help-ph 9606475.

[12] R. Cameron et al., Phys. Rev. D 47, (1993) 3707.

[13] D. Balakov et al. Nucl. Phys. B (Proc. Suppl.) 35 (1994) 180.

[14] W. R. Bardeen, S. H. H. Tye, Phys. Lett. 74B 229 (1978);

T. W. Donnelly et al., Phys. Rev. D 181607 (1978).

[15] B. De Tollis, Nuovo Cimento 35 (1965) 1182;

B. De Tollis, Nuovo Cimento 32 (1964) 757.

[16] Y. Sakurayama, H. Salecker, F.C. Simm, In Istanbul 1989, Proceedings, New frontiers in quantum electrodynamics and quantum optics 285-319.

[17] D. A. Williams et al., Phys. Rev. D 381365 (1988). 